\title{
Protocol for a randomised controlled feasibility trial of exercise rehabilitation for people with postural tachycardia syndrome: the PULSE study
}

Gordon McGregor ${ }^{1,2,3^{*}}$ DD, Siew Wan Hee ${ }^{4}$, Helen Eftekhari ${ }^{5}$, Nikki Holliday ${ }^{2}$, Gemma Pearce ${ }^{2}$, Harbinder Sandhu ${ }^{3}$, Jane Simmonds ${ }^{6}$, Shivam Joshi ${ }^{7}$, Lesley Kavi ${ }^{8}$, Julie Bruce ${ }^{3}$, Sandeep Panikker ${ }^{5}$, Boon Lim ${ }^{9}$ and Sajad Hayat ${ }^{10}$

\begin{abstract}
Background: Postural orthostatic tachycardia syndrome (POTS) is an autonomic nervous system disorder causing an abnormal cardiovascular response to upright posture. It affects around $0.2 \%$ of the population, most commonly women aged 13 to 50 years. POTS can be debilitating; prolonged episodes of pre-syncope and fatigue can severely affect activities of daily living and health-related quality of life (HRQoL). Medical treatment is limited and not supported by randomised controlled trial (RCT) evidence. Lifestyle interventions are first-line treatment, including increased fluid and salt intake, compression tights and isometric counter-pressure manoeuvres to prevent fainting. Observational studies and small RCTs suggest exercise training may improve symptoms and HRQoL in POTS, but evidence quality is low.

Methods: Sixty-two people (aged 18-40 years) with a confirmed diagnosis of POTS will be invited to enrol on a feasibility RCT with embedded qualitative study. The primary outcome will be feasibility; process-related measures will include the number of people eligible, recruited, randomised and withdrawn, along with indicators of exercise programme adherence and acceptability. Secondary physiological, clinical and health-related outcomes including sub-maximal recumbent bike exercise test, active stand test and HRQoL will be measured at 4 and 7 months postrandomisation by researchers blinded to treatment allocation. The PostUraL tachycardia Syndrome Exercise (PULSE) intervention consists of (1) individual assessment; (2) 12-week, once to twice-weekly, supervised out-patient exercise training; (3) behavioural and motivational support; and (4) guided lifestyle physical activity. The control intervention will be best-practice usual care with a single 30-min, one-to-one practitioner appointment, and general advice on safe and effective physical activity. For the embedded qualitative study, participants ( $n=10$ intervention, $n=10$ control) will be interviewed at baseline and 4 months post-randomisation to assess acceptability and the feasibility of progressing to a definitive trial.

(Continued on next page)
\end{abstract}

\footnotetext{
* Correspondence: gordon.mcgregor@coventry.ac.uk

'Department of Cardiopulmonary Rehabilitation, Centre for Exercise \& Health,

University Hospitals Coventry \& Warwickshire NHS Trust, Watch Close,

Coventry CV1 3LN, UK

${ }^{2}$ Centre for Sport, Exercise and Life Sciences, Coventry University, Coventry,

UK

Full list of author information is available at the end of the article
}

(c) The Author(s). 2020 Open Access This article is licensed under a Creative Commons Attribution 4.0 International License, which permits use, sharing, adaptation, distribution and reproduction in any medium or format, as long as you give appropriate credit to the original author(s) and the source, provide a link to the Creative Commons licence, and indicate if changes were made. The images or other third party material in this article are included in the article's Creative Commons licence, unless indicated otherwise in a credit line to the material. If material is not included in the article's Creative Commons licence and your intended use is not permitted by statutory regulation or exceeds the permitted use, you will need to obtain permission directly from the copyright holder. To view a copy of this licence, visit http://creativecommons.org/licenses/by/4.0/ The Creative Commons Public Domain Dedication waiver (http://creativecommons.org/publicdomain/zero/1.0/) applies to the data made available in this article, unless otherwise stated in a credit line to the data. 
(Continued from previous page)

Discussion: There is very little high-quality research investigating exercise rehabilitation for people with POTS. The PULSE study will be the first randomised trial to assess the feasibility of conducting a definitive multicentre RCT testing supervised exercise rehabilitation with behavioural and motivational support, compared to best-practice usual care, for people with POTS.

Trial registration: ISRCTN45323485 registered on 7 April 2020.

Keywords: Postural orthostatic tachycardia syndrome, Exercise, Rehabilitation, Dysautonomia, Cardiac rehabilitation, Randomised controlled trial, Complex intervention, Feasibility

\section{Background}

Postural orthostatic tachycardia syndrome (POTS) affects the autonomic nervous system resulting in an abnormal cardiovascular response to upright posture. It is defined as a clinical syndrome that is usually characterised by (1) frequent symptoms that occur with standing such as light-headedness, palpitations, tremulousness, generalised weakness, blurred vision, exercise intolerance and fatigue; (2) an increase in heart rate of $\geq 30$ beats per minute when moving from a recumbent to a standing position held for more than $30 \mathrm{~s}$; and (3) the absence of orthostatic hypotension (> $20 \mathrm{mmHg}$ drop in systolic blood pressure) [1]. The pathophysiology of POTS is multi-factorial. Symptoms may result from a multitude of mechanisms, including but not limited to volume depletion, immune dysfunction or autoimmune disease, cardiac and physical deconditioning and the inability of lower limb vasoconstriction to compensate for postural blood volume shifts [2]. A disproportionate autonomic response, aimed at rectifying haemodynamic compromise, results in the hallmark features of prolonged pre-syncope (feeling of being about to faint) and fatigue $[1,3]$.

POTS can be debilitating; simple activities may result in persistent orthostatic intolerance. This can lead to poor concentration, palpitations, nausea, 'brain fog' and exercise intolerance [3] affecting activities of daily living and health-related quality of life (HRQoL) [4]. Hypermobility spectrum disorders (HSD) are a common comorbidity [5]. The ability to attend education, undertake gainful employment and care for dependants can be substantially compromised by POTS. A constellation of symptoms can initiate a negative feedback loop by which enforced inactivity further precipitates orthostatic intolerance, immobility and deconditioning $[6,7]$.

Treatment for POTS is limited; medical therapies have not been tested in randomised controlled trials (RCTs) [8]. According to expert consensus, non-pharmacological therapy is first-line treatment [1]. Conservative measures include increased fluid and salt intake, compression tights, isometric counter-pressure manoeuvres (e.g. hand gripping and leg crossing/tensing) to prevent fainting, and psychological interventions to help manage chronic illness [1]. Exercise training is also advocated as an essential component of POTS treatment [1]. However, this is based on only two small studies, one of which excluded people with HSD [9] and one which investigated orthostatic intolerance in military recruits [10]. These studies demonstrated some physiological improvement (increased left ventricular mass/blood volume and decreased standing heart rate), reduced symptoms and improved HRQoL. More recently, a prospective observational study $(n=251)$ enrolled people with POTS on 3 months of unsupervised community exercise [8]. A total of 103 people completed the treatment, of which 73 no longer met the diagnostic criteria for POTS, and HRQoL was improved. However, the study was limited by the lack of a control group, and poor individualisation and supervision of exercise, leading to $60 \%$ attrition.

\section{Rationale for a trial}

Supervised exercise rehabilitation may be an effective therapy for POTS, with resultant clinical and psychosocial benefit. Observational studies, whilst not definitive, suggest that symptoms and psychosocial morbidity may improve [8]. Based on this preliminary evidence, it is important to investigate whether or not people with POTS can benefit from supervised exercise rehabilitation. The broad spectrum of physical ability, symptoms and comorbidities requires that this be initially undertaken within well-designed clinical trials that take account of previous research limitations. The first step is to investigate the feasibility of conducting a multicentre RCT testing a comprehensive exercise rehabilitation intervention, compared to best-practice usual care, for people with POTS.

\section{Methods/design}

\section{Aim}

The aim of the PostUraL tachycardia Syndrome Exercise (PULSE) study is to assess the feasibility of conducting a multi-centre RCT testing a supervised exercise rehabilitation intervention with behavioural and motivational support (PULSE intervention) compared to best-practice usual care for people with POTS.

\section{Objectives}

Undertake a feasibility RCT: Sixty-two participants ( $n$ $=31$ intervention, $n=31$ control) will be randomised to 
the PULSE intervention or control. Process-related measures will include eligibility, recruitment, uptake and intervention adherence. Physiological, clinical and health-related measures will include a sub-maximal graded recumbent bike exercise test, active stand test and HRQoL.

Conduct a qualitative analysis: To explore perceptions, opinions, acceptability and experiences of trial procedures, the PULSE intervention and outcome measures, interviews will be conducted with a sample of participants, drop-outs, and those who declined participation.

\section{Study design and setting}

PULSE is a two-arm feasibility RCT with an embedded qualitative study (Fig. 1). Recruiting simultaneously from two NHS POTS clinics in England (University Hospitals Coventry \& Warwickshire (UHCW) NHS Trust and Imperial College Healthcare NHS Trust), participants will be randomised to the intervention or control arms in a ratio of 1:1, stratified by NHS Trust. Table 1 summarises study methods and design in accordance with the World Health Organization (WHO) Trial Registration Data Set.

\section{Eligibility criteria}

People with POTS meeting the study inclusion criteria are eligible to participate (Table 2).

\section{Participant identification, recruitment and informed consent}

Physicians, arrhythmia nurse specialists or other clinical staff involved in the care of people with POTS will identify eligible participants from two sources: (1) specialist nurse/medical POTS out-patient clinics, (2) local secondary care disease registers. Participant information leaflets will be provided in person during an out-patient clinic appointment or will be sent in the post with a request to return an expression of interest form. Once interest and willingness to be contacted have been confirmed, participants will be contacted by a member of their clinical team and invited to attend a baseline assessment appointment, at which eligibility will be reassessed and informed consent taken. The PULSE study requires different levels of consent: (1) to take part in the trial; (2) for medical notes to be reviewed by designated individuals and personal identifiable information to be securely stored; and (3) to allow telephone contact between the trial team and the participant or designated next of kin.

\section{Randomisation, allocation concealment and blinding}

After all baseline assessments have been completed, participants will be randomised on a 1:1 basis to intervention or control, stratified by centre (UHCW NHS Trust or Imperial
College Healthcare NHS Trust) (Fig. 2). Randomisation will be performed, and allocation concealment maintained, using an online validated randomisation sequence generator, as part of an electronic data capture system (Castor). Only PULSE practitioners, who will be delivering either the control or active interventions, will be informed of group allocation, and only after all baseline measures are complete. Participants and practitioners cannot be blinded to group allocation. PULSE practitioners will deliver both the active and control interventions, so will be aware of treatment allocation after randomisation. Outcome assessment staff, the chief investigator $(\mathrm{CI})$ and the trial statistician will be blinded to group allocation, until statistical analysis is complete.

\section{Interventions}

\section{The PULSE intervention}

To ensure applicability and acceptability further to receipt of study grant funding, the intervention was refined and finalised using co-production methods, in accordance with INVOLVE [13]. A detailed account of this process will be available in an intervention development publication. Briefly, two workshops with patients, public and stakeholders were led by experienced facilitators. During the first workshop, proposed outcome measures and the PULSE intervention were presented to the group and discussed from all stakeholders' perspectives. Interim work was then undertaken by the research team to refine the intervention components and outcome measures prior to workshop two during which the protocol was finalised and implementation and delivery were discussed.

POTS patients and stakeholders (family, nursing, psychology, exercise physiology, physiotherapy, general practice, POTS UK) attended the meetings to ensure that contributions were correctly interpreted [14]. Coproduction techniques allowed these multiple stakeholders to work towards a common goal [13]. It was a flexible and adaptable process, using multiple communication strategies, to ensure inclusion of people who were affected differently by POTS. The process was iterative, with each session informing the next, until a solution was achieved. Researcher notes were analysed thematically, thus informing the refinement and implementation of the PULSE intervention and study [15].

Format: To ensure external validity to the NHS outpatient/community outreach setting, the PULSE intervention is based on existing UK cardio-pulmonary rehabilitation guidelines and service design [16-18].

Programme design: Participants randomised to the PULSE intervention will either (1) access existing cardiopulmonary rehabilitation programmes, i.e. they will 


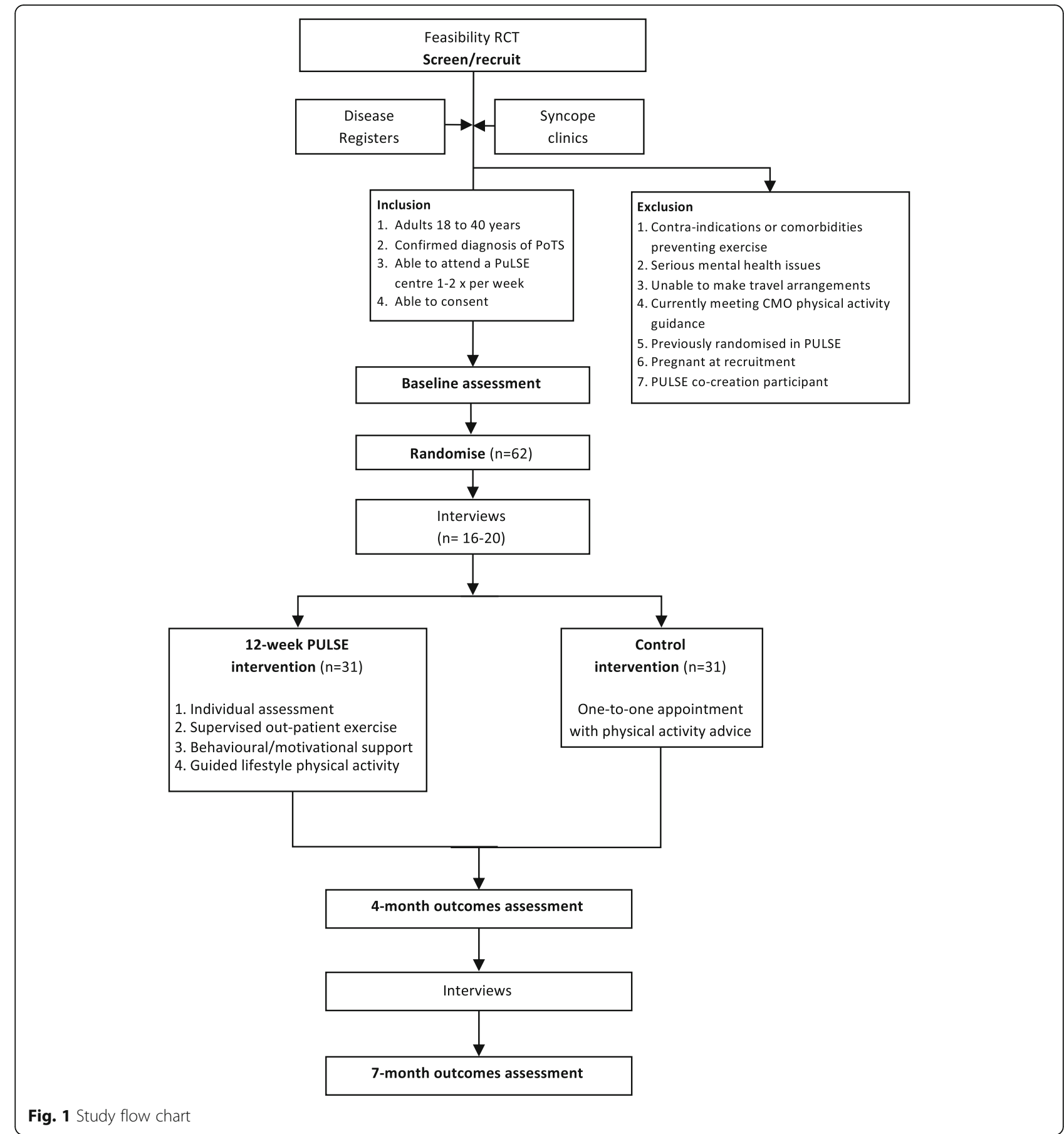

exercise with people with a range of cardiopulmonary conditions, as well as other trial participants randomised to the PULSE intervention, or (2) they will attend dedicated sessions for people with POTS. To encourage peer support, every effort will be made to ensure there are at least two to three PULSE participants exercising at the same centre at the same time. The PULSE intervention has four components:
Component 1: individual assessment and exercise familiarisation

Individual assessment: Participants will undergo a 1-h, one-to-one appointment with a PULSE 'practitioner' (clinical exercise physiologist), to assess and record medical and physical activity history and medication and to discuss goals, expectations and any concerns. 
Table 1 World Health Organization trial registration data set

\begin{tabular}{|c|c|}
\hline Data category & Information \\
\hline $\begin{array}{l}\text { Primary registry and trial } \\
\text { identifying number }\end{array}$ & ISRCTN45323485 \\
\hline $\begin{array}{l}\text { Date of registration in primary } \\
\text { registry }\end{array}$ & 7 April 2020 \\
\hline Secondary identifying numbers & $\begin{array}{l}\text { REC reference: 20/EM/0077 } \\
\text { BHF reference: PG/19/22/34203 }\end{array}$ \\
\hline $\begin{array}{l}\text { Source(s) of monetary or material } \\
\text { support }\end{array}$ & British Heart Foundation Project Grant \\
\hline Primary sponsor & $\begin{array}{l}\text { UHCW NHS Trust } \\
\text { University Hospital } \\
\text { Clifford Bridge Rd., Coventry CV2 2DX } \\
\text { Tel: } 02476966195 \\
\text { Email: R\&DSponsorship@uhcw.nhs.uk }\end{array}$ \\
\hline Secondary sponsor(s) & $\begin{array}{l}\text { Coventry University } \\
\text { Richard Crossman Building } \\
\text { Jordon Well } \\
\text { Coventry University } \\
\text { CV1 5RW } \\
\text { Email: cdu141@coventry.ac.uk }\end{array}$ \\
\hline Contact for public queries & $\begin{array}{l}\text { Coventry University } \\
\text { Richard Crossman Building } \\
\text { Jordon Well } \\
\text { Coventry University } \\
\text { CV1 5RW } \\
\text { Email: cdu141@coventry.ac.uk }\end{array}$ \\
\hline Contact for scientific queries & $\begin{array}{l}\text { Dr Gordon McGregor } \\
\text { Coventry University/UHCW NHS Trust } \\
\text { Tel: } 02476150285 \\
\text { Email: gordon.mcgregor@coventry.ac.uk }\end{array}$ \\
\hline
\end{tabular}

Public title

Scientific title

Supervised exercise rehabilitation for people with postural tachycardia syndrome

Countries of recruitment

PostUraL tachycardia Syndrome Exercise (PULSE): a randomised controlled feasibility study

Health condition(s) or problem(s) Postural orthostatic tachycardia syndrome

studied

Intervention(s)

Intervention group: (1) individual assessment, (2) supervised out-patient exercise programme, (3) behavioural and motivational support, (4) guided lifestyle physical activity.

Control intervention: best-practice usual care

Key inclusion and exclusion criteria

Inclusion: adults (18-40 years) with confirmed diagnosis of POTS and attending syncope out-patient clinics; able to attend a PULSE centre; able to provide informed consent.

Exclusion: absolute contraindications to exercise; currently achieving CMO physical activity guidelines; mental health issue preventing engagement with trial procedures; pregnant at time of recruitment; previous randomisation in PULSE; unable to attend a PULSE centre; took part in PULSE intervention co-creation.

Study type

Type: feasibility, interventional, two-centre

Allocation: randomised

Assignment: parallel

Masking: outcomes assessors, chief investigator, statistician

Date of first enrolment

TBC

Target sample size

62

Recruitment status

Primary outcome(s)

Ready to start recruitment (on hold due to Covid-19)

Feasibility and process indicators: number of patients screened, eligible, recruited, randomised, withdrawn and retained; adherence to exercise rehabilitation programme; length of time to complete each outcome assessment and the whole outcome assessment appointment; willingness of participants to join non-POTS specific exercise rehabilitation programmes; physiological, clinical, patient-reported outcomes to identify a primary outcome for a definitive trial; acceptability of the interventions and the trial (qualitative interviews)

Key secondary outcomes

At 4 and 7 months: exercise capacity — graded recumbent cycle ergometer test; autonomic function —increase in heart rate from supine to 10-min stand; symptom burden-COMPASS 31 dysautonomia scale and fatigue severity scale; HRQoL_EQ-5D-5L; self-efficacy—general self-efficacy scale; exercise tolerability—continuous heart rate monitoring and symptoms during exercise; adverse events.

At baseline and 7 months: semi-structured interviews with participants 
Table 2 Inclusion and exclusion criteria

\begin{tabular}{ll}
\hline Inclusion criteria & Exclusion criteria \\
\hline - Adults 18 to 40 years of age & - Absolute contraindications to \\
- Confirmed diagnosis of POTS [1] & exercise as per international clinical \\
and currently attending specialist & guidelines [11, 12] \\
out-patient clinics & - Any serious mental health/ \\
- Able to attend a PULSE centre 1- & cognitive issue that will prevent \\
2 times/week for 8-12 weeks for & engagement with study \\
exercise training & procedures or increase the risk of \\
- Able to provide informed & exercise complications \\
consent & - Unable to make suitable travel \\
& arrangements \\
& - Currently undertaking structured \\
& exercise/physical activity equivalent \\
& to the Chief Medical Officer (CMO) \\
& guidelines (150 min moderate \\
& exercise per week or 60 min \\
& vigorous per week) \\
& - Previous randomisation in the \\
& present trial \\
& - Pregnancy \\
& - Taken part in co-creation work- \\
& shops to design the PULSE inter- \\
& vention/study
\end{tabular}

Exercise prescription: There are no accepted guidelines for exercise training in POTS. The intervention will be individualised and is based on existing evidence, patient and public involvement (PPI) and co-production sessions and the centres' expertise in the provision of exercise for clinical populations. As such, the PULSE practitioner will prescribe a safe and effective individualised exercise programme $[11,17]$ based on clinical information, data from graded, sub-maximal cycle ergometry and active stand tests and patient-centred goal setting.

Familiarisation sessions: Exercise instruction will be provided on an individual basis during two one-on-one familiarisation sessions in the first week of the programme, and reinforced throughout, by PULSE practitioners and clinical staff. Familiarisation sessions, conducted within the cardiopulmonary rehabilitation programmes, will enable participants to build confidence, whilst PULSE practitioners refine and optimise the exercise prescription. Behavioural and motivational support will also be introduced during these sessions.

\section{Component 2: supervised out-patient exercise programmes}

For the first 6 (of 12) weeks, exercise will be undertaken during one to two sessions per week solely in a controlled gym environment with carefully staged progression in response to participant tolerance and symptoms. For those that are able, moderate intensity dynamic cardiovascular exercise will be prescribed. In addition, 'functional fitness training' will aim to improve orthostatic tolerance and general musculoskeletal deconditioning. This type of training is targeted specifically at the components of physical fitness required for activities of daily living, making use of multi-plane motion, to improve agility, coordination, proprioception, balance and functional strength.

Prescribed exercise: Exercise will be versatile and individualised, incorporating cardiovascular and functional resistance training components. Interval or continuous cardiovascular exercise will be performed at a tolerable intensity (regulated with rating of perceived exertion [RPE] and symptoms) for a manageable time, focusing initially on exercises in the recumbent or semirecumbent position (e.g. rowing machine, recumbent cycle ergometer) to minimise orthostatic tachycardia. Gentle warm-up and cool down will be performed in the manner best suited to individual symptoms. Exercise intensity and time will be gradually increased, and upright exercise introduced as tolerated.

\section{Component 3: behavioural and motivational support}

Comprehensive behavioural change and motivational strategies to improve adherence and compliance to exercise will be incorporated into the PULSE intervention. Every second week, before or after their supervised exercise session, participants will receive a one-to-one, 30min behavioural and motivational session delivered by a trained PULSE practitioner, with the aim of improving short- and long-term adherence to exercise.

The PULSE intervention will be based on a cognitive behavioural framework including self-efficacy and selfmanagement principles with behaviour change theory [19]. In particular, the COM-B model of behaviour change ('capability', 'opportunity', 'motivation' and 'behaviour') will be applied to the content and delivery of the behavioural sessions. Capability relates to increasing confidence through supervised exercise practice and exploring participants' own health beliefs and perceived barriers which may prevent them from progressing with the intervention. For example, quite often people with POTS may avoid certain activities and movement due to a fear of aggravating symptoms [20]. Opportunity, both internal and external to the individual, will be explored through beliefs and thoughts in relation to knowledge and acceptance of POTS, including principles of self-management and skills of living with what is sometimes termed 'the invisible condition'. Access to material and information covered in the sessions will be ensured by producing a participant handbook to allow consolidation of learning and reflection in between sessions. Motivation will also be key to sustained behaviour change for people with POTS. This will be addressed with planning, goal setting and exploration of expectations, with opportunity for self-reflection and discussion of progress, setbacks and overcoming 


\begin{tabular}{|c|c|c|c|c|}
\hline \multirow[b]{3}{*}{ TIMEPOINT } & \multicolumn{4}{|c|}{ STUDY PERIOD } \\
\hline & \multirow{2}{*}{$\begin{array}{c}\text { Enrolment } \\
-t_{1}\end{array}$} & \multirow{2}{*}{$\begin{array}{c}\text { Allocation } \\
0\end{array}$} & \multicolumn{2}{|c|}{ Post-allocation } \\
\hline & & & $\begin{array}{c}t_{1} \\
4 \text { months }\end{array}$ & $\begin{array}{c}t_{2} \\
7 \text { months }\end{array}$ \\
\hline \multicolumn{5}{|l|}{ ENROLMENT: } \\
\hline \multirow{4}{*}{$\begin{array}{r}\text { Eligibility screen } \\
\text { Informed consent } \\
\text { Randomisation } \\
\text { Allocation }\end{array}$} & $\mathrm{X}$ & & & \\
\hline & $\mathrm{X}$ & & & \\
\hline & 62 & $X$ & & \\
\hline & & $\mathrm{X}$ & & \\
\hline \multicolumn{5}{|l|}{ INTERVENTIONS: } \\
\hline \multirow{2}{*}{$\begin{array}{r}\text { PULSE } \\
\text { Usual care }\end{array}$} & 31 & $\bullet$ & $\rightarrow$ & \\
\hline & 31 & 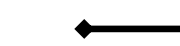 & $\rightarrow$ & \\
\hline \multicolumn{5}{|l|}{ ASSESSMENTS: } \\
\hline $\begin{array}{r}\text { Feasibility and } \\
\text { process measures }\end{array}$ & & $\mathrm{X}$ & $\mathrm{X}$ & $\mathrm{X}$ \\
\hline $\begin{array}{r}\text { Graded recumbent } \\
\text { cycle test }\end{array}$ & & $\mathrm{X}$ & $\mathrm{X}$ & $\mathrm{X}$ \\
\hline & & $X$ & $X$ & $X$ \\
\hline$E Q-5 D-5 L$ & & $\mathrm{X}$ & $\mathrm{X}$ & $\mathrm{X}$ \\
\hline $\begin{array}{r}\text { Generalised self- } \\
\text { efficacy scale }\end{array}$ & & $\mathrm{X}$ & $\mathrm{X}$ & $X$ \\
\hline Fatigue severity scale & & $\mathrm{X}$ & $\mathrm{X}$ & $\mathrm{X}$ \\
\hline Exercise symptoms & & $X$ & $X$ & $X$ \\
\hline Adverse events & & $\mathrm{X}$ & $\mathrm{X}$ & $\mathrm{X}$ \\
\hline $\begin{array}{r}\text { Semi-structured } \\
\text { interviews }\end{array}$ & & $\mathrm{X}$ & & $X$ \\
\hline
\end{tabular}

Fig. 2 Schedule of enrolment, interventions and assessments

obstacles [21]. This will be combined with the theory of planned behaviour $[22,23]$ to explore participant's perceived control, intention and motivation to adhere to the PULSE intervention.

The PULSE practitioners will be trained to use open questions and motivational interviewing to assess participants' current beliefs and encourage behaviour change. In accordance with existing literature, it will be important to manage expectations. Previous work in POTS has indicated that symptoms may worsen for $4-6$ weeks before a lasting benefit is gained [21].

\section{Component 4: lifestyle physical activity}

Half-way through the supervised exercise intervention, thus after 6 weeks of centre-based exercise has been safely completed, and/or individual exercise tolerance has been evaluated, participants will be encouraged to undertake self-directed, lifestyle physical activity at home in addition to the supervised sessions. This will help ensure that exercise is performed every other day. Activities such as swimming, walking and cycling will be encouraged for those in whom it is appropriate. 


\section{Control intervention: best practice usual care}

Participants in the control arm will be provided with freely available advice on lifestyle physical activity (POTS UK website) [24] during a one-to-one session, lasting approximately $30 \mathrm{~min}$, with a PULSE practitioner. They will not receive any further input during the control intervention period. Participants will be permitted to continue with any current physical activity but will not receive supervised exercise training or behavioural and motivational sessions from PULSE practitioners.

\section{Safety}

The PULSE intervention will be delivered in cardiopulmonary rehabilitation units with qualified staff and appropriate emergency equipment. Lifestyle physical activity will be lower intensity and replicate exercises performed in the gym under supervision. Intervention practitioners will be clinical exercise physiologists, experienced in assessment, prescription and delivery of exercise in clinical populations and trained in the standardised delivery of the PULSE intervention. Additional training in exercise modification to avoid joint subluxation and dislocation in hypermobile participants will be provided by a specialist physiotherapist.

\section{Primary outcome}

The primary outcome will be overall feasibility and process-related measures:

- Number of patients screened, eligible, recruited, randomised, withdrawn and retained.

- Adherence to exercise rehabilitation programme-number of supervised exercise and lifestyle physical activity sessions completed over 12 weeks.

- Length of time to complete each outcome measure and the whole outcome assessment appointment.

- Willingness of participants to join non-POTS specific exercise rehabilitation programmes (i.e. standard cardiopulmonary rehabilitation programmes). Participant preference will be recorded, and uptake to each programme model monitored.

- Physiological, clinical, patient-reported outcomes to confirm/identify a primary outcome for a definitive trial.

- Acceptability of the interventions and the trial (embedded qualitative study).

\section{Secondary outcomes}

Physiological, clinical and patient-reported outcomes will be assessed during study visits at baseline, and postintervention at 4 and 7 months post-randomisation (Table 2).
Exercise capacity will be measured with a graded submaximal recumbent cycle ergometer assessment [11]. This will be a simple test (maximum $10 \mathrm{~min}$ ) involving gradual increments in workload every minute. Workload increments and test termination will be determined by symptoms, heart rate, blood pressure and RPE responses which will be recorded throughout. The increase in heart rate from supine to 10-min stand test (active stand test) will be conducted as per clinical practice [25]. This is a commonly used diagnostic test for POTS, measuring the heart rate response to $10 \mathrm{~min}$ (or however long is manageable dependant on symptoms) of standing. It will be performed only as an outcome measure in this trial, not for diagnostic purposes.

The symptom burden of POTS will be measured with the Composite Autonomic Symptom Score (COMPASS 31) dysautonomia scale [26]. This is a self-rating questionnaire evaluating six domains of autonomic function: orthostatic intolerance, vasomotor, secretomotor, gastrointestinal, bladder and pupillomotor domains. In addition to domain scores, an overall total score from 0 to 100 can be computed, with a higher score indicating a greater disease burden. Health-related quality of life will be evaluated with the EQ-5D-5L [27], a validated, generic HRQoL measure consisting of five dimensions, each with five levels of response. It has good test-retest reliability, is simple to use and gives a single preferencebased index value for health status. The Fatigue Severity Scale [28], a validated nine-item questionnaire, will be used to assess disabling fatigue. Each item is rated on a seven-point scale, from strongly disagree to strongly agree. A total score is derived from all nine questions; a higher score indicates a greater impact of fatigue on everyday activities. Self-efficacy will be measured with the generalised self-efficacy scale [29] which is a 10-item psychometric scale designed to assess optimistic selfbeliefs to cope with a variety of difficult demands in life. A single score between 10 and 40 is generated; greater self-efficacy is represented by a higher score.

To evaluate the participants' response to exercise sessions and assessments, any POTS-related or associated symptoms will be recorded. Adverse and serious adverse events will be documented and reported to the trial management group (TMG) in accordance with the principles of good clinical practice (GCP).

\section{Sample size}

This is a feasibility RCT to assess recruitment, uptake, adherence, acceptability and clinical, physiological and health-related outcomes. Thus, the sample size is not based on a power calculation. Nevertheless, the aim is to estimate any possible effect of the change of heart rate from supine to 10-min stand between the two treatment arms to inform a future definitive RCT. The sample size 
is determined using the confidence interval approach to provide a given level of precision. From a single-arm study investigating a 3-month community exercise programme in a similar population [8], the mean increase in heart rate from supine to 10-min stand at baseline was $46 \pm 17 \mathrm{bpm}$ and post-intervention was $23 \pm 14$ $\mathrm{bpm}$. For the purpose of this trial, the standard deviation (SD) is assumed to be the same for both treatment arms, and the SD of the difference of the change is $17 \mathrm{bpm}$. Therefore, the total sample size required to obtain a $95 \%$ confidence interval width of $20 \mathrm{bpm}$ is 46 participants [30]. Assuming a drop-out rate of $25 \%$, the total sample size required is 62 participants (31 per treatment arm). Participants will be randomised equally to the intervention or usual care arms (i.e. 1:1 ratio).

\section{Data analysis}

Participant demographics and physiological, clinical and patient-reported outcomes will be summarised by treatment arms as mean and SD, median and interquartile range (for continuous data) or frequency and percentage (for categorical data) at baseline, and 4 and 7 months post-randomisation. The primary outcome is feasibility; therefore, process indicators relating to recruitment and intervention delivery will be assessed. Descriptive statistics will be presented by recruitment centre and treatment arm. The difference in outcomes between treatment arms at 4 and 7 months post-randomisation and the difference of the change from baseline to 4 months post-randomisation between arms will be computed. All estimates will be reported with a 95\% confidence interval. No formal hypothesis testing will be performed.

All data will be analysed and reported in accordance with the CONSORT statement. All primary analyses are planned on an intention-to-treat basis (i.e. data will be analysed according to the treatment arm the participant was originally allocated to, irrespective of what treatment they actually received) with a secondary per protocol analysis.

\section{Embedded qualitative study}

Semi-structured interviews, conducted by an experienced qualitative researcher, with approximately $n=10$ intervention group and $n=10$ usual care, at baseline and 4 months post-randomisation (same participants at both time-points), will be digitally recorded, pseudonymised and transcribed verbatim. Data will be analysed using the framework method [31], broadly as follows:

- Data familiarisation: reading of complete interview transcripts, listening to original audio recordings and use of field notes;
- Identifying a thematic framework: key issues, concepts and themes will be identified, and an index of codes developed;

- Indexing: the index generated through identification of the thematic framework will be applied to all data;

- Charting: a summary of each passage of text will be transferred into a chart to allow more overall and abstract consideration of index codes across the data set and by each individual;

- Mapping and interpretation: understanding the meaning of key themes, dimensions and the broad overall picture of the data and identifying and understanding the typical associations between themes and dimensions.

The charting process will provide an opportunity to code data from numerous perspectives. The computer package NVivo will be used to organise the analysis. The findings of the qualitative analyses will be reported as a separate chapter in the final report but will also be incorporated in the discussion to bring together a synthesis of all the results, thus helping to explore and explain the overall 'value' of the interventions. Quantitative and qualitative data will be integrated using a mixed methods matrix where quantitative responses can be compared to interview data and recorded on a matrix [32].

\section{Transition to a definitive trial}

The ADePT (A Process for Decision-making after Pilot and Feasibility Trials) framework [33] will be used to identify and examine issues and problems methodically, and to appraise and find appropriate solutions to inform the decision-making process to transition from this twocentre feasibility trial to a definitive multi-centre RCT.

\section{Data collection and management}

Personal data collected during the study will be handled and stored in accordance with General Data Protection Regulation (GDPR). Personal identifying information will be stored at each site for follow-up purposes. Disclosure of confidential information will only be considered if there is an issue which may jeopardise the safety of the participant or another person, in accordance with local standard operating procedures (SOPs) and the UK regulatory framework. An online validated, GCP compliant, electronic data capture system (Castor) will be used by researchers at each site to record and store study data. All data will be pseudonymised after the collection of baseline demographics for each participant. Participants will be assigned a unique study ID, which will be used to identify all documents associated with that participant for the duration of the study. In line with participant consent, direct access to source data/documents will be 
granted to authorised representatives from the sponsor, host institution and the regulatory authorities, to permit study-related monitoring, audits and inspections.

\section{Trial monitoring}

The trial management group (TMG), chaired by the CI, and consisting of project management staff, lay representatives, co-investigators, the trial statistician and sponsor representatives, will meet monthly for the duration of the project. For this small feasibility RCT, there will be no independent data monitoring committee or trial steering committee; these functions will be completed by the TMG.

\section{Adverse event management}

An adverse event (AE) will be defined as any untoward medical occurrence involving a participant, which does not necessarily have a causal relationship with the intervention or trial. Expected AEs for the PULSE study that will be recorded but not reported include pre-syncope, muscle and joint stiffness, soreness and dislocation and tiredness and fatigue. Any unexpected AEs related to PULSE will be recorded and reported to the TMG. Serious adverse events (SAEs) that have no causal relationship with PULSE will not be reported to oversight committees. Causality and expectedness will be confirmed by the CI with clinician support. SAEs deemed to be unexpected and possibly, probably or definitely related to PULSE will be notified to the Research Ethics Committee (REC) within 15 days. All AEs and SAEs will be recorded within $24 \mathrm{~h}$ of the investigator being made aware.

\section{Patient and public involvement}

The PULSE intervention components were initially developed based on existing evidence, previous clinical work at our centres and extensive patient and public involvement (PPI). Eighteen people with POTS participated in teleconference consultation prior to the grant funding application. The intervention was further developed and refined during two co-creation workshops with multiple stakeholders (as outlined in more detail above). Lay representatives will sit on the TMG and will be invited to contribute to dissemination and reporting on completion of the study.

\section{Discussion}

Postural orthostatic tachycardia syndrome can be a debilitating condition, with pre-syncope and fatigue, amongst other symptoms, significantly impacting on activities of daily living and HRQoL. Whilst prevalence is estimated to be between 0.2 and $1 \%$, it is increasingly recognised that POTS is commonly misdiagnosed, thus prevalence is likely to be much higher. In the absence of effective and appropriately tested medical and pharmacological therapies, lifestyle interventions have the potential to improve symptoms and help manage this longterm condition.

Exercise rehabilitation is not routinely offered to people with POTS as it has not been evaluated in well-designed clinical trials. Preliminary reports from non-randomised controlled studies indicate that exercise rehabilitation programmes, as provided almost universally for people with long-term cardiac and pulmonary conditions, may be beneficial for people with POTS. The PULSE study will be the first to assess the feasibility of an exercise intervention with behavioural and motivational support for people with POTS, to inform whether or not a future multi-centre RCT investigating clinical and cost-effectiveness can be delivered in the NHS. The feasibility of a supervised exercise programme, a behavioural and motivational support intervention, and a number of important clinical, physiological and patient-reported outcome measures, will be evaluated within the framework of a feasibility RCT. If feasibility is proven, a definitive multi-centre RCT should be conducted in the future.

\section{Trial status}

All approvals are in place, and the trial is ready to begin recruitment. Currently, all trial activities have been temporarily suspended due to the COVID-19 pandemic.

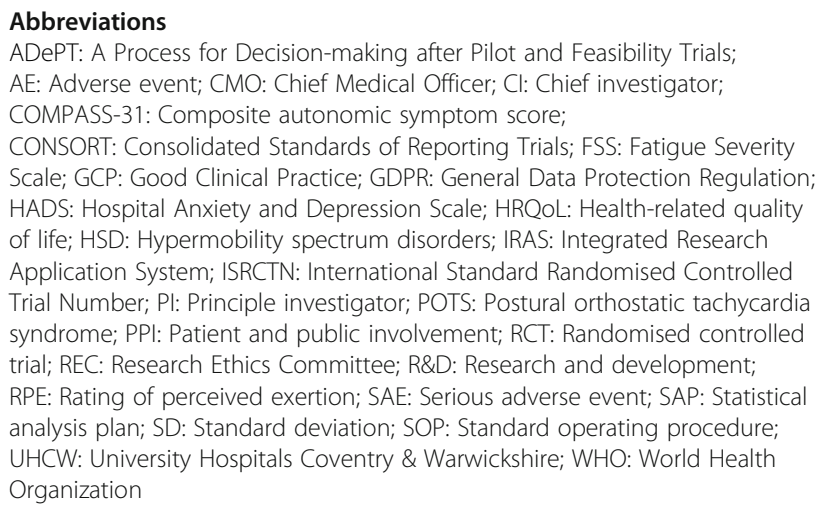

\section{Acknowledgements}

The authors would like to thank our lay partners and all the stakeholders who took part in the co-creation of the PULSE intervention and trial. We would also like to thank trial implementation, management and support teams at UHCW NHS Trust and Coventry University, R\&D and cardiac rehabilitation teams at each site and all trial participants.

\section{Authors' contributions}

GM is the chief investigator for the study and obtained funding with support of SWH, HE, NH, GP, HS, LK, BL, SP, JS, JB and SH. All contributed to study design. GM, SWH, JB, (clinical trials); SWH (statistics); HS, GP (health psychology); NH, GP (co-creation, qualitative); GM, JS (clinical exercise physiology/physiotherapy); HE (specialist nursing); and LK, BL, SP, SH (medical) provided expertise in their respective discipline and authored the relevant section of the protocol and manuscript. GM prepared the manuscript which all authors edited before approving the final version. 


\section{Funding}

This study is funded by a British Heart Foundation (BHF) Project Grant: PG/ $19 / 22 / 34203$. The views expressed are those of the authors and not necessarily those of the BHF. JB is supported by National Institute for Health Research (NIHR) Research Capability Funding via UHCW NHS Trust.

\section{Availability of data and materials}

The datasets used and/or analysed during the current study will be available from the corresponding author on reasonable request.

\section{Ethics approval and consent to participate}

Ethical approval was received via the Integrated Research Application System (266145) and the East Midlands, Nottingham Research Ethics Committee (20/ EM/0077) and Health Research Authority on 3 April 2020. Each study site will confirm local NHS Trust Research \& Development (R\&D) department capacity and capability prior to commencing recruitment. All participants will provide written informed consent before participating in the study. UHCW NHS Trust and Coventry University are co-sponsors for the trial. The trial will be conducted in accordance with the declaration of Helsinki.

\section{Consent for publication}

Not applicable

\section{Competing interests}

None

\section{Author details}

'Department of Cardiopulmonary Rehabilitation, Centre for Exercise \& Health, University Hospitals Coventry \& Warwickshire NHS Trust, Watch Close, Coventry CV1 3LN, UK. ${ }^{2}$ Centre for Sport, Exercise and Life Sciences, Coventry University, Coventry, UK. ${ }^{3}$ Warwick Clinical Trials Unit, Warwick Medical School, University of Warwick, Coventry, UK. ${ }^{4}$ Division of Health Sciences, Warwick Medical School, University of Warwick, Coventry, UK. ${ }^{5}$ Department of Cardiology, University Hospitals Coventry \& Warwickshire NHS Trust, Coventry, UK. ${ }^{6}$ UCL Great Ormond Street Institute of Child Health, Faculty of Population Health, University College London, London, UK. ${ }^{7}$ Research \& Development, University Hospitals Coventry \& Warwickshire NHS Trust, Coventry, UK. ${ }^{8}$ POTS UK, Birmingham, UK. ${ }^{9}$ Department of Cardiology, Imperial College Healthcare NHS Trust, London, UK. ${ }^{10}$ Department of Cardiology, Hamad Medical Corporation, Doha, Qatar.

Received: 28 April 2020 Accepted: 12 October 2020

Published online: 19 October 2020

\section{References}

1. Sheldon RS, Grubb BP 2nd, Olshansky B, Shen WK, Calkins H, Brignole M, et al. 2015 heart rhythm society expert consensus statement on the diagnosis and treatment of postural tachycardia syndrome, inappropriate sinus tachycardia, and vasovagal syncope. Heart Rhythm. 2015;12(6):e41-63.

2. Freeman $R$, Wieling W, Axelrod FB, Benditt DG, Benarroch E, Biaggioni I, et al. Consensus statement on the definition of orthostatic hypotension, neurally mediated syncope and the postural tachycardia syndrome. Clin Auton Res. 2011;21(2):69-72.

3. Karas B, Grubb BP, Boehm K, Kip K. The postural orthostatic tachycardia syndrome: a potentially treatable cause of chronic fatigue, exercise intolerance, and cognitive impairment in adolescents. Pacing Clin Electrophysiol. 2000;23(3):344-51.

4. McDonald C, Koshi S, Busner L, Kavi L, Newton JL. Postural tachycardia syndrome is associated with significant symptoms and functional impairment predominantly affecting young women: a UK perspective. BMJ Open. 2014;4(6):e004127.

5. Hakim A, O'Callaghan C, De Wandele I, Stiles L, Pocinki A, Rowe P. Cardiovascular autonomic dysfunction in Ehlers-Danlos syndrome-hypermobile type. Am J Med Genet C: Semin Med Genet. 2017;175(1):168-74.

6. Raj SR. Postural tachycardia syndrome (POTS). Circulation. 2013;127(23): 2336-42.

7. Wells R, Spurrier AJ, Linz D, Gallagher C, Mahajan R, Sanders P, et al. Postural tachycardia syndrome: current perspectives. Vasc Health Risk Manag. 2018; $14: 1-11$

8. George SA, Bivens TB, Howden EJ, Saleem Y, Galbreath MM, Hendrickson D, et al. The international POTS registry: evaluating the efficacy of an exercise training intervention in a community setting. Heart Rhythm. 2016:13(4):943-50.

9. Fu Q, Vangundy TB, Galbreath MM, Shibata S, Jain M, Hastings $J$, et al. Cardiac origins of the postural orthostatic tachycardia syndrome. J Am Coll Cardiol. 2010;55(25):2858-68

10. Winker R, Barth A, Bidmon D, Ponocny I, Weber M, Mayr O, et al. Endurance exercise training in orthostatic intolerance: a randomized, controlled trial. Hypertension. 2005;45(3):391-8.

11. ACSM. Guidelines for exercise testing and prescription. 10th ed. Riverwoods: Lippincott Williams \& Wilkins; 2017.

12. Whaley M, Brubaker $P$, Otto R, Armstrong L. American College of Sports Medicine's guide to clinical exercise testing and prescription. Philadelphia: Lippincott Williams \& Wilkins; 2013.

13. Hickey G, Brearley S, Coldham T, Denegri S, Green G, Staniszewska S, Tembo D, Torok K, Turner K. Guidance on co-producing a research project. Sothampton: INVOLVE; 2018

14. Malagon-Maldonado G. Qualitative research in health design. HERD. 2014; 7(4):120-34.

15. Green JT, N. Qualitative methods for health research. London: Sage; 2004

16. Bolton CE, Bevan-Smith EF, Blakey JD, Crowe P, Elkin SL, Garrod R, et al. British Thoracic Society guideline on pulmonary rehabilitation in adults. Thorax. 2013;68(Suppl 2):ii1-30.

17. ACPICR. Standards for physical activity and exercise in the cardiac population 2015. Available at: http://acpicr.com

18. Buckley JP, Furze G, Doherty P, Speck L, Connolly S, Hinton S, et al. BACPR scientific statement: British standards and core components for cardiovascular disease prevention and rehabilitation. Heart. 2013;99(15): 1069-71.

19. Bandura A. Self-efficacy: toward a unifying theory of behavioral change. Psychol Rev. 1977;84(2):191-215.

20. Stiles LE, Cinnamon J, Balan I. The patient perspective: what postural orthostatic tachycardia syndrome patients want physicians to know. Auton Neurosci. 2018:215:121-5.

21. Michie S, van Stralen MM, West R. The behaviour change wheel: a new method for characterising and designing behaviour change interventions. Implement Sci. 2011;6:42.

22. Ajzen I. The theory of planned behavior. Organ Behav Hum Decis Process. 1991;50(2):179-211.

23. Ajzen I, Fishbein M. Understanding attitudes and predicting social behavior. Englewood Cliffs: Prentice-Hall; 1980.

24. POTS UK. Exercise examples https://www.potsuk.org/exercise examples2020.

25. Bryarly M, Phillips LT, Fu Q, Vernino S, Levine BD. Postural orthostatic tachycardia syndrome. J Am Coll Cardiol. 2019;73(10):1207.

26. Sletten DM, Suarez GA, Low PA, Mandrekar J, Singer W. COMPASS 31: a refined and abbreviated composite autonomic symptom score. Mayo Clin Proc. 2012:87(12):1196-201.

27. Herdman M, Gudex C, Lloyd A, Janssen M, Kind P, Parkin D, et al. Development and preliminary testing of the new five-level version of EQ-5D (EQ-5D-5L). Qual Life Res. 2011;20(10):1727-36.

28. Krupp LB, LaRocca NG, Muir-Nash J, Steinberg AD. The fatique severity scale. Application to patients with multiple sclerosis and systemic lupus erythematosus. Arch Neurol. 1989;46(10):1121-3.

29. Schwarzer RJ, M. Measures in health psychology: a user's portfolio. Causal and control beliefs. Windsor: NFER-NELSON; 1995.

30. Machin DCM, Tan SB, Tan SH. Sample size tables for clinical studies. Chapter 10 confidence interval. Chichester: Wiley-Blackwell; 2009.

31. Gale NK, Heath G, Cameron E, Rashid S, Redwood S. Using the framework method for the analysis of qualitative data in multi-disciplinary health research. BMC Med Res Methodol. 2013:13:117.

32. Mars T, Ellard D, Carnes D, Homer K, Underwood M, Taylor SJ. Fidelity in complex behaviour change interventions: a standardised approach to evaluate intervention integrity. BMJ Open. 2013;3(11):e003555.

33. Bugge C, Williams B, Hagen S, Logan J, Glazener C, Pringle S, et al. A process for Decision-making after Pilot and feasibility Trials (ADePT): development following a feasibility study of a complex intervention for pelvic organ prolapse. Trials. 2013;14:353.

\section{Publisher's Note}

Springer Nature remains neutral with regard to jurisdictional claims in published maps and institutional affiliations. 